\section{P055 CXCL13 AS BIOMARKER FOR HISTOLOGICAL INVOLVEMENT IN SJOGREN'S SYNDROME}

${ }^{1} S$ Colafrancesco*, ${ }^{1} R$ Priori, ${ }^{2} \mathrm{C}$ Smith, ${ }^{1} \mathrm{~A}$ Minniti, ${ }^{2} \mathrm{~V}$ lannizzotto, ${ }^{2} \mathrm{E}$ Pipi, ${ }^{2} \mathrm{~S}$ Nayar, ${ }^{2} \mathrm{~J}$ Campos, ${ }^{3} \mathrm{D}$ Lucchesi, ${ }^{1} \mathrm{~F}$ Arienzo, ${ }^{4} \mathrm{~B}$ Cerbelli, ${ }^{4} \mathrm{C}$ Giordano, ${ }^{1} \mathrm{G}$ Valesini, ${ }^{3} \mathrm{M}$ Bombardieri, 2,5BA Fisher, ${ }^{2,5} \mathrm{~F}$ Barone. 'Department of Internal Medicine and Medical Specialties, Sapienza Università di Roma, Rome, Italy; ${ }^{2}$ Institute of Inflammation and Ageing, University of Birmingham, Birmingham; ${ }^{3}$ Centre for Experimental Medicine and Rheumatology, Queen Mary University of London, London, UK; ${ }^{4}$ Department of Radiological, Oncological and Pathological Sciences, Sapienza Università di Roma, Rome, Italy; ${ }^{5}$ National Institute for Health Research (NIHR) Birmingham Biomedical Research Centre, University of Birmingham, Birmingham, UK

\subsection{6/annrheumdis-2018-EWRR2019.47}

Career situation of first and presenting author Post-doctoral fellow.

Introduction Sjogren's syndrome (SS) is an autoimmune condition characterised by systemic B cell activation, autoantibody production and ectopic germinal centers (GC) formation within salivary gland (SG). The extent of SG infiltrate has been proposed as biomarker of disease severity. Plasma levels of CXCL13 correlate with GC activity in animal models and disease severity in SS, suggesting its potential use as a surrogate serum marker to monitor local $\mathrm{B}$ cell activation.

Objectives To evaluate the potential role of CXCL13 as biomarker of SG pathology in two independent SS cohorts.

Methods 109 patients with SS were recruited at Sapienza University of Rome (Italy) $(n=60)$, or at Queen Elizabeth Hospital in Birmingham and Barts Health NHS Trust in London $(n=49)$. Both sera and matched paraffin-embedded minor SG biopsy were available. Sicca $(n=57)$ and healthy subjects (HS) $(n=19)$ sera were used as control. CXCL13 gene expression was also assessed in 25 frozen SGs.

Results CXCL13 serum levels were higher in SS patients [90.3 (84.2) $\mathrm{pg} / \mathrm{ml}$, compared to both sicca $[61.9$ (38.6) $\mathrm{pg} / \mathrm{ml}$ ), $\mathrm{p}=0.0005]$ and HS [36.5 (40.18) pg/ml, p<0.0001]. No differences in average CXCL13 levels were detected between the two SS cohorts [83 (95.2) $\mathrm{pg} / \mathrm{ml}$ and 103 (64.9) $\mathrm{pg} / \mathrm{ml}$, respectively]. In both Italian and British cohorts, serum levels of CXCL13 correlated with the percentage of SG infiltration $(p=0.0008$ and $p=0.0004$, respectively), FS $(p=0.0010$ and $\mathrm{p}=0.0103$, respectively) and mean foci area $(\mathrm{p}=0.0167$ and $\mathrm{p}=0.0054$, respectively); higher serum levels were observed in patients with segregated foci $(p=0.0553$ and $p=0.0019$, respectively) and GCs ( $\mathrm{p}=0.0147$ and $\mathrm{p}=0.0044$, respectively). Higher CXCL13 serum levels were also associated with antiRo/SSA antibodies $(p=0.0007$, Italian cohort and $p=0.0294$, British cohort) as well as rheumatoid factor (RF) $(p=0.0037$, Italian Cohort and $\mathrm{p}=0.0033$, British Cohort). Tissue expression of CXCL13 was moderately correlate with the mean foci area and percentage of infiltration. Higher expression of CXCL13 transcripts was observed in SGs with segregated foci $(p=0.0057)$ and GCs $(p=0.0162)$. Serum levels of CXCL13 did not correlate with local mRNA levels of the same cytokine.

Conclusions Our data foster the use of CXCL13 to monitor the extent of local pathology in SS and its validation in longitudinal clinical studies. Both serum and tissue expression of CXCL13 correlate with SS histological severity, suggesting a major role of this chemokine in SG lymphocytes recruitment and organization.

Disclosure of Interest None declared.

\section{P056 A NOVEL PGC-1B/NFATC-1 PATHWAY IN MONOCYTES FACILITATES OSTEOCLASTOGENESIS IN RHEUMATOID ARTHRITIS}

J-D Ma, J Jing, Y-Q Mo, Li Q-H, J-Z Lin, L-F Chen, L Dai*. Department of Rheumatology, Sun Yat-Sen Memorial Hospital, Sun Yat-Sen University, Guangzhou, China

\subsection{6/annrheumdis-2018-EWRR2019.48}

Career situation of first and presenting author Resident.

Introduction The underlying mechanism of excessive osteoclastogenesis causing bone erosion in rheumatoid arthritis (RA) remains elusive. PGC-1 $\beta$ is implicated in transcriptional regulation of osteoclastogenesis but its role in RA pathogenesis is unknown.

Objectives To investigate whether PGC-1 $\beta$ regulated osteoclastogenesis in RA.

Methods PGC-1 $\beta$ expression in peripheral CD14+ monocytes from RA patients were detected by immunofluorescence, flow cytometry and western blot. Peripheral CD14+ monocytes from RA patients or healthy controls were transfected with lentivirus for PGC-1 $\beta$ gene silencing or over-expression and cultured with M-CSF and RANKL. Mature osteoclasts and their bone resorption activity were determined by TRAP, Factin and toluidine blue staining. DC-STAMP and bone degrading enzymes as well as signaling molecules were detected by western blot.

Results

1. Increased nuclear accumulation of PGC-1 $\beta$ was observed in peripheral CD14+ monocytes from RA patients and their relative PGC-1 $\beta$ protein expression was higher than that in healthy controls. Cultured with RANKL and M-CSF, the cell counts of mature osteoclasts on day 14 and 21 and the pit area of bone resorption lacunae on day 21 were significantly higher in RA patients than those in healthy controls.

2. PGC-1 $\beta$ knockdown in monocytes suppressed the expression of cathepsin K, TRAP and MMP-9 as well as osteoclast differentiation and bone resorption activity, while PGC-1 $\beta$ over-expression markedly promoted these indicators and osteoclastogenesis. Furtherly, over-expressed PGC-1 $\beta$ increased the nuclear expression of NFATc-1. VIVIT, inhibitor of NFATc-1 activation, limited the effect of over-expressed PGC-1 $\beta$ on promoting the expression of cathepsin K, TRAP and MMP-9 in peripheral CD14+ monocytes from healthy controls.

3. ChIP-QPCR analysis confirmed the immunoprecipitation of PGC-1 $\beta$ and the NFATc-1 promoter which indicated that PGC-1 $\beta$ binds to the NFATc-1 promoter region. Dualluciferase reporter gene assay showed that over-expressed PGC-1 $\beta$ on the peripheral CD14+ monocytes from healthy controls increased the transcriptional activity of NFATc- 1 in a dose-dependent manner.

Conclusions Our data revealed a novel PGC-1 $\beta /$ NFATc-1 pathway contributing to excessive osteoclastogenesis in RA, which implied a potential therapeutic target of PGC-1 $\beta$ for osteoclast inhibition in RA.

Acknowledgements This work was supported by National Natural Science Foundation of China (81471597, 81671612 and 81601427) and Guangdong Natural Science Foundation (2017A030313576 and 2017A030310236).

Disclosure of Interest None declared. 